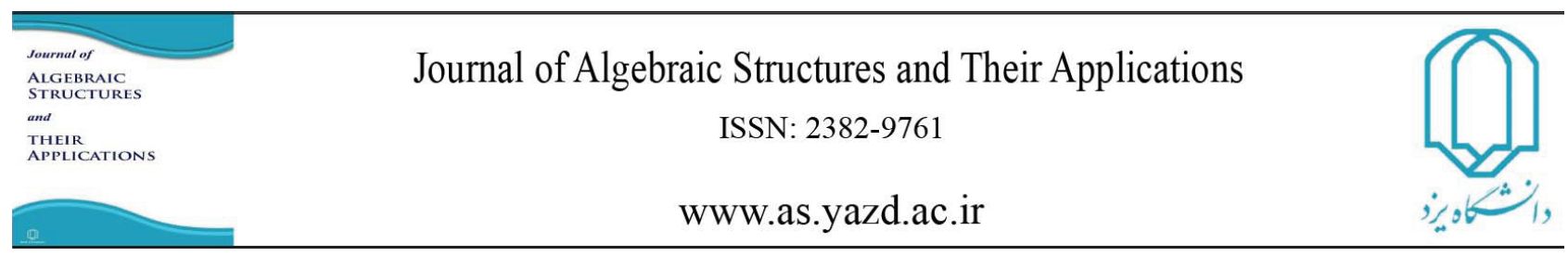

Algebraic Structures and Their Applications Vol. 5 No. 1 ( 2018 ), pp 51-68.

\title{
BOOLEAN CENTER OF LATTICE ORDERED EQ-ALGEBRAS WITH BOTTOM ELEMENT
}

\author{
NEDA MOHTASHAMNIA AND LIDA TORKZADEH*
}

Communicated by B. Davvaz

\begin{abstract}
In this paper, some new properties of $E Q$-algebras are investigated. We introduce and study the notion of Boolean center of lattice ordered $E Q$-algebras with bottom element. We show that in a good $\ell E Q$-algebra $E$ with bottom element the complement of an element is unique. Furthermore, Boolean elements of a good bounded lattice $E Q$-algebra are characterized. Finally, we obtain conditions under which Boolean center of an $E Q$-algebra $E$ is the subalgebra of $E$.
\end{abstract}

\section{INTRODUCTION}

In order to deal with uncertainty and inaccurate reasoning, an accurate type of logic such as fuzzy logic can be used. The algebraic properties and features of the structure of the truth values, uniquely determine any many-valued logic. It is widespread approved that in fuzzy logic, there must be a residuated lattice, which will probably fulfill some extra properties. Now we will distinguish different types of formal fuzzy logics. Residuated lattice based fuzzy logic is a well-known fuzzy logic example of which include Łukasiewicz logic [1], $M T L$-logic [4], Gödel logic [5], $B L$-logic [8] and $R_{0}$-logic [9], etc. The algebraic semantics that correspond to these

DOI:http://dx.doi.org/10.29252/asta.5.1.51

MSC(2010): Primary: 03G05.

Keywords: $E Q$-algebra, blEQ-algebra, Boolean element.

Received: 24 December 2017, Accepted: 20 September 2018

*Corresponding author 
logics are $M V$-algebras, $M T L$-algebras, $B L$-algebras and $R_{0}$-algebras, respectively [11].

$E Q$-algebras were introduced by $\mathrm{V}$. Novák and B. De Baets with the intent to generalize residuated lattice [10].

An $E Q$-algebra has three binary operations (meet, multiplication and a fuzzy equality) and a top element. A binary operation implication is derived from fuzzy equality by $x \rightarrow y=$ $(x \wedge y) \sim x$. This implication and multiplication are not closely tied by the adjunction. Special classes of $E Q$-algebras have been studied in $[2,3]$. Moreover the $\{\wedge, \rightarrow, 1\}$-reduct of good $E Q$-algebras are $B C K$-meet-semilattices.

Our main aim is to find interesting properties of Boolean elements in a lattice $E Q$-algebra with bottom element. The set of these elements is called Boolean center and we show that it is a subalgebra of $E$.

This paper is organized as follows: in section 2, we review the basic definitions, special types of $E Q$-algebras and their properties. In section 3, we investigate some new properties of $E Q$ algebras. Section 4 is dedicated to showing that a complement of an element may not be unique unless in a special type of $E Q$-algebra. Finally, we determine the set of all Boolean elements of $E$ is the universe of a Boolean subalgebra of $E$.

1.1. Preliminaries. We recall the basic definitions and results of $E Q$-algebras and we put in the evidence many rules of calculus

An algebra $\xi=(E, \wedge, \otimes, \sim, 1)$ of type $(2,2,2,0)$ is called an $E Q$-algebra where for all $a, b, c, d \in E$ the following hold:

(E1) $(E, \wedge, 1)$ is a $\wedge$-semilattice with the ordering $a \leq b$ iff $a \wedge b=a$ and top element 1 ,

$(E 2)(E, \otimes, 1)$ is a monoid and $\otimes$ is isotone in both arguments w.r.t. $a \leq b$,

(E3) $a \sim a=1$, (reflexivity axiom)

$(E 4)((a \wedge b) \sim c) \otimes(d \sim a) \leq c \sim(d \wedge b)$, (substitution axiom)

$(E 5)(a \sim b) \otimes(c \sim d) \leq(a \sim c) \sim(b \sim d)$, (congruence axiom)

$(E 6)(a \wedge b \wedge c) \sim a \leq(a \wedge b) \sim a$, (monotonicity axiom)

(E7) $a \otimes b \leq a \sim b .[3]$

The binary operations $\wedge, \otimes$ and $\sim$ are called meet, multiplication and a fuzzy equality, respectively. Clearly, $(E, \leq)$ is a partial order. We denote, for $a, b \in E$

$$
\widetilde{a}:=a \sim 1 \text { and } a \rightarrow b:=(a \wedge b) \sim a
$$

The binary operation $\rightarrow$ will be called implication. If 0 is a bottom element of $\mathrm{E}, \mathrm{E}$ is denoted $b E Q$-algebra, then we may define the unary operation $\neg$ on E, for all $a \in E$, by $\neg a=a \sim 0$.

For $a, b \in E$ and $n \in N$, we define $a \rightarrow^{0} b=b, a \rightarrow^{n+1} b=a \rightarrow\left(a \rightarrow^{n} b\right)$. If $a=1$, 
$a \rightarrow^{n+1} b$ is denoted by $\widetilde{b}^{n+1}$.

Lemma 1.1. [3, 7] Let $\xi$ be an EQ-algebra. Then the following properties hold for all $a, b, c, d \in$ E:

$\left(e_{1}\right) a \sim b=b \sim a$,

$\left(e_{2}\right)(a \sim b) \otimes(b \sim c) \leq(a \sim c)$,

$\left(e_{3}\right)(a \rightarrow b) \otimes(b \rightarrow c) \leq(a \rightarrow c)$ and $(b \rightarrow c) \otimes(a \rightarrow b) \leq(a \rightarrow c)$,

$\left(e_{4}\right) a \sim d \leq(a \wedge b) \sim(d \wedge b)$,

$\left(e_{5}\right) a \otimes b \leq a \wedge b \leq a, b$,

$\left(e_{6}\right) b \leq \widetilde{b} \leq a \rightarrow b$,

(e $e_{7}$ If $a \leq b$, then $a \rightarrow b=1, b \rightarrow a=a \sim b, \widetilde{a} \leq \widetilde{b}, c \rightarrow a \leq c \rightarrow b$ and

$b \rightarrow c \leq a \rightarrow c$,

(es) If $a \leq b \leq c$, then $a \sim c \leq a \sim b$ and $a \sim c \leq b \sim c$,

$\left(e_{9}\right) a \otimes(a \sim b) \leq \widetilde{b}$,

$\left(e_{10}\right)$ If $a \leq b \rightarrow c(a \leq b \sim c)$, then $a \otimes b \leq \widetilde{c}$,

$\left(e_{11}\right)(a \rightarrow b) \otimes(c \rightarrow d) \leq(a \wedge c) \rightarrow(b \wedge d)$,

$\left(e_{12}\right)(a \rightarrow c) \otimes(b \rightarrow c) \leq(a \wedge b) \rightarrow c$,

$\left(e_{13}\right)(c \rightarrow a) \otimes(c \rightarrow b) \leq c \rightarrow(a \wedge b)$,

$\left(e_{14}\right) a \rightarrow d \leq(b \rightarrow a) \rightarrow(b \rightarrow d)$,

$\left(e_{15}\right) a \rightarrow d \leq(d \rightarrow b) \rightarrow(a \rightarrow b)$,

$\left(e_{16}\right)((a \wedge b) \rightarrow c) \otimes(d \rightarrow a) \leq(d \wedge b) \rightarrow c$,

$\left(e_{17}\right) a \rightarrow(b \rightarrow c) \leq b \rightarrow(a \rightarrow \widetilde{c})$,

$\left(e_{18}\right) a \rightarrow(b \rightarrow c) \leq b \rightarrow(a \rightarrow(a \rightarrow c))$,

$\left(e_{19}\right) a \rightarrow(b \rightarrow c) \leq(a \otimes b) \rightarrow \widetilde{c}^{4}$,

$\left(e_{20}\right)(b \rightarrow a) \otimes(a \rightarrow b) \leq a \sim b \leq(b \rightarrow a) \wedge(a \rightarrow b)$.

Definition 1.2. [11,2] Let $\xi$ be an EQ-algebra. We say that it is

(i) good, if for all $a \in E, \widetilde{a}=1 \rightarrow a=a$,

(ii) separated, if for all $a, b \in E, a \sim b=1$ implies $a=b$,

(iii) semi-separated, if for all $a \in E, a \sim 1=1$ implies $a=1$,

(iv) a lattice-ordered, denoted lEQ-algebra, if the underlying $\wedge$-semilattice is a lattice,

$(v)$ an $\ell E Q$-algebra, if it is a lattice-ordered EQ-algebra and for all $a, b, c, d \in E,((a \vee b) \sim$

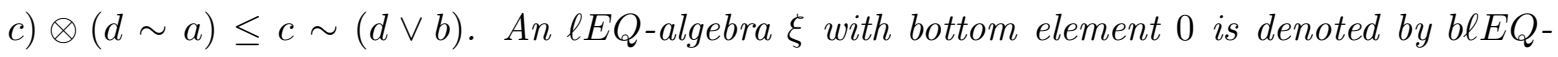
algebra,

(vi) prelinear, if for all $a, b \in E, 1$ is the unique upper bound in $E$ of the set $\{(a \rightarrow b),(b \rightarrow a)\}$, (vii) spanned, if it contains a bottom element 0 and $\widetilde{0}=0$. 
It is clear that by $\left(e_{9}\right)$ every good $E Q$-algebra is a separated $E Q$-algebra and also every separated $E Q$-algebra is a semi-separated $E Q$-algebra.

Theorem 1.3. [3] The following properties are provable in all good EQ-algebras $\xi$, for all $a, b, c \in E$ :

$\left(g_{1}\right) a \leq(a \rightarrow b) \rightarrow b$,

$\left(g_{2}\right) a \leq b \rightarrow c$ iff $b \leq a \rightarrow c$,

$\left(g_{3}\right) a \rightarrow(b \rightarrow c) \leq(a \otimes b) \rightarrow c$ and $a \rightarrow(b \rightarrow c) \leq(b \otimes a) \rightarrow c$,

$\left(g_{4}\right) a \rightarrow(b \rightarrow c)=b \rightarrow(a \rightarrow c)$ (Exchange principle),

$\left(g_{5}\right) a \leq b \rightarrow c$ implies $a \otimes b \leq c$ and $b \otimes a \leq c$,

$\left(g_{6}\right)$ If $\xi$ is a lEQ-algebra, then $(a \vee b) \rightarrow c=(a \rightarrow c) \wedge(b \rightarrow c)$,

$\left(g_{7}\right)$ If $E$ has a bottom element 0 , then $a \leq \neg \neg a$.

Theorem 1.4. $[3,11]$ Let $\xi$ be an $\ell E Q$-algebra. Then the following hold for all $a, b, c \in E$ :

$\left(l_{1}\right) a \rightarrow b=(a \wedge b) \sim a=(a \vee b) \sim b=(a \vee b) \rightarrow b$,

$\left(l_{2}\right)(a \rightarrow c) \otimes(b \rightarrow c) \leq(a \vee b) \rightarrow c$,

$\left(l_{3}\right)(a \sim b) \otimes(c \sim d) \leq(a \vee c) \sim(b \vee d)$,

$\left(l_{4}\right) a \rightarrow b \leq(a \vee c) \rightarrow(b \vee c)$,

$\left(l_{5}\right) a \sim b \leq(a \vee c) \sim(b \vee c)$

Lemma 1.5. [3] The following statements are equivalent in any EQ-algebra:

(i) $\xi$ is separated,

(ii) $a \leq b$ iff $a \rightarrow b=1$, for all $a, b \in E$.

Definition 1.6. [3] A nonempty subset $F$ of an EQ-algebra $\xi$ is called a prefilter of $\xi$, if for all $a, b \in E$, the following conditions hold

$\left(P F_{1}\right) 1 \in F$,

$\left(P F_{2}\right)$ If $a, a \rightarrow b \in F$, then $b \in F$.

The set of all prefilters of $\mathrm{E}$ is denoted by $\mathrm{PF}(\mathrm{E})$.

For a nonempty subset $X$ of an $E Q$-algebra $\xi$, the smallest prefilter of $\xi$ which contains $X$, i.e. $\bigcap\{F \in P F(E): X \subseteq F\}$, is said to be a prefilter of $\xi$ generated by $X$ and will be denoted by $\langle X>$. In [7], it is shown that

$<X>=\left\{a \in E: x_{1} \rightarrow\left(x_{2} \rightarrow\left(x_{3} \rightarrow \ldots\left(x_{n} \rightarrow a\right) \ldots\right)\right)=1\right.$, for some $x_{i} \in X$ and $\left.n \geq 1\right\}$.

Theorem 1.7. In every EQ-algebra $\xi$ for $a, b \in E$, we have:

(i) $a \leq b$ implies $\langle b>\subseteq<a>$,

(ii) $<a \wedge b>=<a>\vee<b>=<\{a, b\}>$,

(iii) In an $\ell E Q$-algebra $\xi$, we have $\langle a \vee b>=<a>\cap<b>$ and also the lattice $(P F(E) ; \subseteq)$ is a complete Brouwerian lattice [7]. 
Lemma 1.8. [7] An EQ-algebra $\xi$ is semi-separated iff $<1>=\{1\}$.

\section{Some Properties of EQ-ALGEBras}

In this section, we investigate some new properties of $E Q$-algebras.

Theorem 2.1. An EQ-algebra $\xi$ is good iff $x \leq 1 \rightarrow a$ implies $x \leq a$ for all $x, a \in E$.

Proof. Suppose that $x \leq 1 \rightarrow$ implies $x \leq a$ for all $x, a \in E$. Hence for $x \in E$, we have $\widetilde{x}=1 \rightarrow x \leq 1 \rightarrow x$ implies $\widetilde{x} \leq x$. Therefore by $\left(e_{6}\right) \widetilde{x}=x$, for all $x \in E$, that is $\xi$ is good.

The converse is clear by $\left(g_{5}\right)$.

Lemma 2.2. Let $\xi$ be an EQ-algebra. We have the following statements for $a, b, c \in E$ :

$\left(e_{21}\right)(a \rightarrow b) \rightarrow(a \rightarrow c) \leq a \rightarrow(b \rightarrow \widetilde{c})$,

$\left(e_{22}\right) a \leq(a \rightarrow b) \rightarrow \widetilde{b}$

Proof. $\left(e_{21}\right) B y\left(e_{6}\right),\left(e_{7}\right)$ and $\left(e_{17}\right)$ we obtain

$$
\begin{aligned}
b \leq a \rightarrow b & \Rightarrow(a \rightarrow b) \rightarrow \widetilde{c} \leq b \rightarrow \widetilde{c} \\
& \Rightarrow a \rightarrow[(a \rightarrow b) \rightarrow \widetilde{c}] \leq a \rightarrow(b \rightarrow \widetilde{c}) \\
& \Rightarrow(a \rightarrow b) \rightarrow(a \rightarrow c) \leq a \rightarrow[(a \rightarrow b) \rightarrow \widetilde{c}] \leq a \rightarrow(b \rightarrow \widetilde{c}) .
\end{aligned}
$$

$\left(e_{22}\right) B y\left(e_{6}\right)$ and $\left(e_{15}\right)$ we have

$$
a \leq 1 \rightarrow a \leq(a \rightarrow b) \rightarrow(1 \rightarrow b)=(a \rightarrow b) \rightarrow \widetilde{b}
$$

Theorem 2.3. Let $\xi$ be an $l E Q$-algebra and for $a, b \in E, a \vee b=1$. Then

(i) $\widetilde{a} \vee \widetilde{b}=1$,

(ii) $\widetilde{b} \leq a \rightarrow b \leq \widetilde{\widetilde{b}}$

(iii) $(a \rightarrow b) \vee(b \rightarrow a)=1$.

Proof. (i) We have $a \leq \widetilde{a}$ and $b \leq \widetilde{b}$, so $1=a \vee b \leq \widetilde{a} \vee \widetilde{b}$. Thus $\widetilde{a} \vee \widetilde{b}=1$.

(ii) By $\left(e_{6}\right)$ we have $b \leq \widetilde{b} \leq(a \rightarrow b) \rightarrow \widetilde{b}$, so by $\left(e_{22}\right)$ we can say $a, b \leq(a \rightarrow b) \rightarrow \widetilde{b}$.

Since $1=a \vee b \leq[(a \rightarrow b) \rightarrow \widetilde{b}]$, we conclude by $\left(e_{10}\right), a \rightarrow b=1 \otimes(a \rightarrow b) \leq \widetilde{\widetilde{b}}$. So by $\left(e_{6}\right)$, $\widetilde{b} \leq a \rightarrow b \leq \widetilde{\widetilde{b}}$

(iii) It is clear by $(i)$ and $($ ii).

Let $\xi$ be an $E Q$-algebra with bottom element 0 . Then $\neg a \rightarrow(a \rightarrow 0)=1$ implies that $0 \in\langle a\rangle \vee\langle\neg a>$ for all $a \in E$, so $\langle a\rangle \vee\langle\neg a\rangle=E$. The following lemma shows that 
$<a>\cap<\neg a>$ may not be 1 , in general.

$\omega$ is the set of nonnegative integers. For $a, z \in E$ and $n \in \omega$ we define $a \rightarrow^{0} z=z, a \rightarrow^{n+1}$ $z=a \rightarrow\left(a \rightarrow^{n} z\right)$. If $a=1, a \rightarrow^{n+1} z$ denoted by $\widetilde{z}^{n+1}$.

Lemma 2.4. Let $\xi$ be an EQ-algebra. Then, the following statements are equivalent, for all $a, b, c \in E:$

(i) $a \rightarrow c=b \rightarrow c=1$ implies $c=1$,

(ii) $<a>\cap<b>=\{1\}$.

Proof. $(i) \Rightarrow($ ii $)$ : Assume $c \in<a>\cap<b>$, there exist positive integers $n_{1}, n_{2}$ such that

$$
a \rightarrow^{n_{1}} c=b \rightarrow^{n_{2}} c=1 \text {. }
$$

Put $n=\max \left\{n_{1}, n_{2}\right\}$, we obtain

$$
a \rightarrow\left(a \rightarrow^{n-1} c\right)=a \rightarrow^{n} c=1 \text { and } b \rightarrow\left(b \rightarrow^{n-1} c\right)=b \rightarrow^{n} c=1 .
$$

Then by applying $\left(e_{10}\right)$ and $\left(e_{17}\right)$ continuously, we can say there is positive integer $k_{1}$ such that

$$
a \leq 1 \rightarrow\left(a \rightarrow^{n-1} c\right) \leq\left(a \rightarrow^{n-1} \widetilde{c}^{k_{1}}\right) .
$$

On the other hand by using $\left(e_{6}\right)$ we have

$$
\widetilde{c}^{k_{1}} \leq b \rightarrow^{n-1} \widetilde{c}^{k_{1}}
$$

Now we get that

$$
a \leq\left(a \rightarrow^{n-1} \widetilde{c}^{k_{1}}\right) \leq a \rightarrow^{n-1}\left(b \rightarrow^{n-1} \widetilde{c}^{k_{1}}\right) .
$$

Since we have $b \rightarrow^{n} c=1$, by using $\left(e_{7}\right),\left(e_{17}\right)$ and $\left(e_{10}\right)$ we can find positive integers $k_{2}, k_{3}$ such that

$$
\begin{aligned}
1=a \rightarrow^{n-1}\left(b \rightarrow^{n} c\right) & \Rightarrow 1=b \rightarrow\left(a \rightarrow^{n-1}\left(b \rightarrow^{n-1} \widetilde{c}^{k_{2}}\right)\right), \\
& \Rightarrow 1 \leq b \rightarrow\left(a \rightarrow^{n-1}\left(b \rightarrow^{n-1} \widetilde{c}^{k_{2}}\right)\right), \\
& \Rightarrow b \leq a \rightarrow^{n-1}\left(b \rightarrow^{n-1} \widetilde{c}^{k_{3}}\right) .
\end{aligned}
$$

Put $k=\max \left\{k_{1}, k_{3}\right\}$, so we obtain

$$
1=a \rightarrow\left(a \rightarrow^{n-1}\left(b \rightarrow^{n-1} \widetilde{c}^{k}\right)\right)=b \rightarrow\left(a \rightarrow^{n-1}\left(b \rightarrow^{n-1} \widetilde{c}^{k}\right)\right) .
$$

Hence by hypothesis, we have $a \rightarrow^{n-1}\left(b \rightarrow^{n-1} \widetilde{c}^{k}\right)=1$. Clearly, iterating the process we can obtain there is positive integer $l$ such that

$$
b \rightarrow^{n-1} \widetilde{c}^{l}=a^{0} \rightarrow\left(b \rightarrow^{n-1} \widetilde{c}^{l}\right)=1 .
$$

Similarly, we can obtain $a \rightarrow^{n-1} \widetilde{c}^{l}=1$. Repeating the above procedure $n-1$ times, there exists positive integer $p$ such that

$$
a \rightarrow \widetilde{c}^{p}=b \rightarrow \widetilde{c}^{p}=1 .
$$

Hence by hypothesis we obtain $\widetilde{c^{p}}=1$ and by $\left(e_{6}\right)$ we get that

$$
1=\widetilde{c}^{p} \leq a \rightarrow \widetilde{c}^{p-1} \text { and } 1=\widetilde{c}^{p} \leq b \rightarrow \widetilde{c}^{p-1}
$$


Now we get that $\widetilde{c}^{p-2}=1$. Therefore by continuing this way we get that $c=1$ and so $<a>\cap<b>=\{1\}$.

$($ ii $) \Rightarrow($ i): Let $a \rightarrow c=b \rightarrow c=1$. So we obtain $c \in<a>\cap<b>=\{1\}$.

Consider $E=\{0, a, b, c, d, 1\}$ such that $0<a, b<c<1$ and $0<b<d<1$. Multiplication and fuzzy equality on $E$ are defined below. It is clear that $(E, \wedge, \otimes, \sim, 1)$ is an $b l E Q$-algebra $[2]$.

\begin{tabular}{|c|c|c|c|c|c|c|c|c|c|c|c|c|c|}
\hline$\otimes$ & 0 & $a$ & $b$ & $c$ & $d$ & 1 \\
\hline 0 & 0 & 0 & 0 & 0 & 0 & 0 \\
\hline$a$ & 0 & $a$ & 0 & $a$ & 0 & $a$ \\
\hline$b$ & 0 & 0 & 0 & 0 & $b$ & $b$ \\
\hline$c$ & 0 & $a$ & 0 & $a$ & $b$ & $c$ \\
\hline$d$ & 0 & 0 & $b$ & $b$ & $d$ & $d$ \\
\hline 1 & 0 & $a$ & $b$ & $c$ & $d$ & 1 \\
\hline$a$ & 1 & $d$ & $c$ & $b$ & $a$ & 0 \\
\hline$b$ & $c$ & $b$ & 1 & $d$ & $c$ & $b$ \\
\hline$c$ & $b$ & $c$ & $d$ & 1 & $b$ & $c$ \\
\hline$d$ & $a$ & 0 & $c$ & $b$ & 1 & $d$ \\
\hline 1 & 0 & $a$ & $b$ & $c$ & $d$ & 1 \\
\hline
\end{tabular}

\begin{tabular}{|c|c|c|c|c|c|c|}
\hline$\rightarrow$ & 0 & $a$ & $b$ & $c$ & $d$ & 1 \\
\hline 0 & 1 & 1 & 1 & 1 & 1 & 1 \\
\hline$a$ & $d$ & 1 & $d$ & 1 & $d$ & 1 \\
\hline$b$ & $c$ & $c$ & 1 & 1 & 1 & 1 \\
\hline$c$ & $b$ & $c$ & $d$ & 1 & $d$ & 1 \\
\hline$d$ & $a$ & $a$ & $c$ & $c$ & 1 & 1 \\
\hline 1 & 0 & $a$ & $b$ & $c$ & $d$ & 1 \\
\hline
\end{tabular}

It is easy to see that $c$ and $d$ satisfy in Lemma $3.4(i)$ and also $\langle c\rangle=\{c, a, 1\}$ and $<d\rangle=$ $\{d, 1\}$. So $<c>\cap<d>=\{1\}$.

\section{Boolean elements in EQ-algebras}

Let $\xi$ be a lattice $E Q$-algebra with bottom element 0 . Recall that an element $a \in E$ is called complemented if there is an element $b \in E$ such that $a \vee b=1$ and $a \wedge b=0$, if such element $b$ exists it is called a complement of $a$. The set of all complemented elements of $a \in E$ is denoted by $B(a)$ and $B(E)=\cup_{a \in E} B(a)$ is called a Boolean center of $E$.

In the sequel a lattice $E Q$-algebra with bottom element is denoted by $b l E Q$-algebra.

We know, in a distributive bounded lattice, complements of an element $a \in E$ are unique. So by [2] in every prelinear and separated $\ell E Q$-algebra with bottom element 0 , complements (if exist) are unique.

The following examples show $E Q$-algebras that their some elements may have more than a complement.

Example 3.1. (i) Consider $E=\{0, a, b, c, d, 1\}$ such that $0<a<b<1$ and $0<c<d<1$. Multiplication and fuzzy equality on $E$ are defined below. 


\begin{tabular}{|c|c|c|c|c|c|c|c|c|c|c|c|c|c|}
\hline$\otimes$ & 0 & $a$ & $b$ & $c$ & $d$ & 1 \\
\hline 0 & 0 & 0 & 0 & 0 & 0 & 0 \\
\hline$a$ & 0 & 0 & 0 & 0 & 0 & $a$ \\
\hline$b$ & 0 & 0 & 0 & 0 & 0 & $b$ \\
\hline$c$ & 0 & 0 & 0 & 0 & 0 & $c$ \\
\hline$d$ & 0 & 0 & 0 & 0 & 0 & $d$ \\
\hline 1 & 0 & $a$ & $b$ & $c$ & $d$ & 1 \\
\hline 0 & 1 & $d$ & $d$ & $d$ & $d$ & 0 \\
\hline$a$ & $d$ & 1 & $d$ & $d$ & $d$ & $b$ \\
\hline$b$ & $d$ & $d$ & 1 & $d$ & $d$ & $b$ \\
\hline$c$ & $d$ & $d$ & $d$ & 1 & $d$ & $d$ \\
\hline$d$ & $d$ & $d$ & $d$ & $d$ & 1 & $d$ \\
\hline 1 & 0 & $b$ & $b$ & $d$ & $d$ & 1 \\
\hline
\end{tabular}

Then $(E, \wedge, \otimes, \sim, 1)$ is a blEQ-algebra and $|B(x)|>1$, for all $x \in E-\{0,1\}$.

(ii) Consider $E=\{0, a, b, c, 1\}$ such that $0<a<b<1$ and $0<c<1$. Multiplication and fuzzy equality on $E$ are defined below.

\begin{tabular}{|c|c|c|c|c|c|}
\hline$\otimes$ & 0 & $a$ & $b$ & $c$ & 1 \\
\hline 0 & 0 & 0 & 0 & 0 & 0 \\
\hline$a$ & 0 & 0 & 0 & 0 & $a$ \\
\hline$b$ & 0 & 0 & 0 & 0 & $b$ \\
\hline$c$ & 0 & 0 & 0 & $c$ & $c$ \\
\hline 1 & 0 & $a$ & $b$ & $c$ & 1 \\
\hline 0 & 0 & $a$ & $b$ & $c$ & 1 \\
\hline$a$ & 1 & 1 & 1 & 1 & 1 \\
\hline$b$ & 1 & 1 & 1 & 1 & 1 \\
\hline$c$ & 1 & 1 & 1 & 1 & 1 \\
\hline 1 & 1 & 1 & 1 & 1 & 1 \\
\hline
\end{tabular}

Then $(E, \wedge, \otimes, \sim, 1)$ is a blEQ-algebra. It is clear $B(c)=\{a, b\}$.

By the following lemma, we can find conditions under which complements of an element $a \in E$ are unique i.e $|B(a)|=1$.

Lemma 3.2. Let $\xi$ be a blEQ-algebra and $a \in E$. We have for $b, c \in B(a)$ :

(i) $b \leq a \rightarrow c$ and $c \leq a \rightarrow b$,

(ii) In an $\ell E Q$-algebra, $c \leq \widetilde{b}$ and $b \leq \widetilde{c}$,

(iii) In a good $\ell E Q$-algebra, $c=b$.

Proof. (i) We have by hypotesis

$$
\begin{aligned}
b & \leq 1 \otimes(1 \rightarrow b), \quad b y\left(e_{6}\right) \\
& =[(a \wedge b) \rightarrow(a \wedge c)] \otimes(1 \rightarrow b) \\
& \leq(a \wedge 1) \rightarrow(a \wedge c), \quad b y\left(e_{16}\right) \\
& \leq a \rightarrow c . \quad b y\left(e_{7}\right)
\end{aligned}
$$

Similarly we conclude $c \leq a \rightarrow b$.

(ii) Let $\xi$ be an $\ell E Q$-algebra. Then by $(i)$ and $\left(l_{4}\right)$ we have

$$
b \leq a \rightarrow c \leq(a \vee c) \rightarrow(c \vee c)=1 \rightarrow c=\widetilde{c} .
$$

Similarly we can obtain $c \leq \widetilde{b}$.

(iii) It is clear by $($ ii). 
Lemma 3.3. Let $\xi$ be a blEQ-algebra and $a \in B(E)$. Then:

(i) $b \leq \neg a$, for all $b \in B(a)$,

(ii) $a \otimes(b \vee c) \leq \widetilde{0}$, for all $b, c \in B(a)$,

(iii) $a \vee \neg a=1$,

(iv) $\widetilde{\neg} \vee \widetilde{a}=1$,

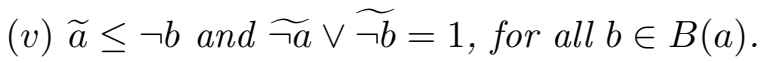

Proof. $(i)$ Let $b \in B(a)$. Then by $\left(e_{6}\right)$ and $\left(e_{13}\right)$, we have:

$$
b \leq a \rightarrow b=(a \rightarrow b) \otimes(a \rightarrow a) \leq a \rightarrow(a \wedge b)=a \rightarrow 0=\neg a .
$$

(ii) By $(i)$, we obtain $(b \vee c) \leq \neg a=a \rightarrow 0$. So, we get that $a \otimes(b \vee c) \leq \widetilde{0}$, by $\left(e_{10}\right)$.

(iii) Let $a \in B(E)$. Then there exists $b \in E$ such that $b \leq \neg a$. So $1=a \vee b \leq a \vee \neg a$, that is $a \vee \neg a=1$.

(iv) Since $a \leq \widetilde{a}$ and $\neg a \leq \widetilde{\neg a}$, so by (iii) we have $1=a \vee \neg a \leq \widetilde{a} \vee \widetilde{\neg a}$. Thus $\widetilde{a} \vee \widetilde{\neg a}=1$.

$(v)$ Let $a \in B(E)$ and $b \in B(a)$. Then $a \vee b=1$ and $a \wedge b=0$. Thus by $\left(e_{6}\right)$ we have

$$
\widetilde{a} \leq b \rightarrow a=(a \wedge b) \sim b=\neg b .
$$

Also we obtain by (iv), $1=\widetilde{a} \vee \widetilde{\neg a} \leq \neg b \vee \widetilde{\neg a} \leq \widetilde{\neg b} \vee \widetilde{\neg a}$, that is $\widetilde{\neg b} \vee \widetilde{\neg a}=1$.

A characterization of $B(E)$ is shown as follows:

Theorem 3.4. In a good blEQ-algebra $\xi, a \in B(E)$ iff $a \vee \neg a=1$.

Proof. Let $a \vee \neg a=1$. Then by $\left(g_{6}\right)$, we have

$$
0=\neg 1=(\neg a \vee a) \rightarrow 0=(\neg a \rightarrow 0) \wedge(a \rightarrow 0)=\neg \neg a \wedge \neg a .
$$

So by $\left(g_{7}\right)$, we obtain $a \wedge \neg a \leq \neg \neg a \wedge \neg a=0$, that is $a \wedge \neg a=0$. Therefore $a \in B(E)$.

The converse follows from Lemma 4.3 part (iii).

By the following example, we show that the condition " oood" $^{\prime \prime}$ in the Theorem 4.4 is necessary.

Example 3.5. Consider $E=\{0, a, b, c, 1\}$ such that $0<a, b<c<1$. Multiplication and fuzzy equality on $E$ are defined below.

\begin{tabular}{|c|c|c|c|c|c|}
\hline$\otimes$ & 0 & $a$ & $b$ & $c$ & 1 \\
\hline 0 & 0 & 0 & 0 & 0 & 0 \\
\hline$a$ & 0 & 0 & 0 & 0 & 0 \\
\hline$b$ & 0 & 0 & 0 & 0 & 0 \\
\hline$c$ & 0 & 0 & 0 & $c$ & 0 \\
\hline 1 & 0 & $a$ & $b$ & $c$ & 1 \\
\hline 0 & 0 & $a$ & $b$ & $c$ & 1 \\
\hline$a$ & 1 & 1 & $a$ & $a$ & $a$ \\
\hline$b$ & $a$ & $a$ & 1 & $c$ & $c$ \\
\hline$c$ & $a$ & $a$ & $c$ & 1 & $c$ \\
\hline 1 & $a$ & $a$ & $c$ & $c$ & 1 \\
\hline
\end{tabular}

Then $(E, \wedge, \otimes, \sim, 1)$ is a blEQ-algebra, while, it is not a good. It is clear that $a \vee \neg a=1$ while $a \notin B(E)$.

Some properties of a Boolean element of separated, good and spanned $b l E Q$-algebra are obtained from the following lemmas. 
Lemma 3.6. Let $\xi$ be a separated blEQ-algebra and $a \in B(E)$. Then for all $b \in E$, we have

(i) $\neg a \rightarrow a=\widetilde{a}$ and $a \rightarrow \neg a=\widetilde{\neg a}$,

(ii) $(a \rightarrow b) \rightarrow a=\widetilde{a}$,

(iii) $\neg \neg a \leq \widetilde{a}$.

Proof. (i) For $a \in B(E)$, by $\left(e_{22}\right)$ we have $\neg a \leq(\neg a \rightarrow a) \rightarrow \widetilde{a}$, and by $\left(e_{6}\right)$ we have $a \leq \widetilde{a} \leq(\neg a \rightarrow a) \rightarrow \widetilde{a}$ and so

$$
1=a \vee \neg a \leq(\neg a \rightarrow a) \rightarrow \widetilde{a} .
$$

Similarly

$$
1=a \vee \neg a \leq(a \rightarrow \neg a) \rightarrow \widetilde{\neg a}
$$

Thus we conclude

$$
(\neg a \rightarrow a) \rightarrow \widetilde{a}=1 \text { and }(a \rightarrow \neg a) \rightarrow \widetilde{\neg a}=1 .
$$

Since $\xi$ is a separated EQ-algebra, by Lemma 2.5 we get that

$$
\neg a \rightarrow a \leq \widetilde{a} \text { and } a \rightarrow \neg a \leq \widetilde{\neg a} .
$$

Also $a, \neg a \leq 1$ implies $\widetilde{a} \leq \neg a \rightarrow a$ and $\widetilde{\neg a} \leq a \rightarrow \neg a$. Therefore $\widetilde{a}=\neg a \rightarrow a$ and $\widetilde{\neg a}=a \rightarrow \neg a$.

(ii) By $\left(e_{7}\right)$ and $(i)$ we obtain

$$
\begin{aligned}
0 \leq b & \Rightarrow \neg a \leq a \rightarrow b \\
& \Rightarrow(a \rightarrow b) \rightarrow a \leq \neg a \rightarrow a=\widetilde{a},
\end{aligned}
$$

On the other hand, we have

$$
a \rightarrow b \leq 1 \Rightarrow \tilde{a}=1 \rightarrow a \leq(a \rightarrow b) \rightarrow a .
$$

Therefore $\widetilde{a}=(a \rightarrow b) \rightarrow a$.

(iii) We have by $\left(e_{7}\right)$ and $(i)$

$$
\begin{aligned}
0 \leq a & \Rightarrow \neg a \rightarrow 0 \leq \neg a \rightarrow a \\
& \Rightarrow \neg \neg a \leq \widetilde{a} .
\end{aligned}
$$

Lemma 3.7. Let $\xi$ be a good blEQ-algebra and $a \in B(E)$. We have the following statements for $b, c \in E$ :

(i) $\neg a \in B(E)$,

(ii) $\neg a \rightarrow a=a, a \rightarrow \neg a=\neg a$ and $(\neg a \rightarrow a) \vee(a \rightarrow \neg a)=1$,

(iii) $a \rightarrow b=a \rightarrow(a \rightarrow b)$,

$(i v) a \rightarrow(b \rightarrow c)=(a \rightarrow b) \rightarrow(a \rightarrow c)$, 
(v) $(a \rightarrow b) \rightarrow b \leq(b \rightarrow a) \rightarrow a$,

(vi) $[(a \rightarrow b) \rightarrow \neg a] \rightarrow \neg a=a \rightarrow \neg \neg b$,

(vii) $\neg \neg a=a$.

Proof. (i) Let $a \in B(E)$. Then by Lemma 4.3, we have $a \vee \neg a=1$ and by the proof of Theorem 4.4 we obtain $a \wedge \neg a=0$, that is $\neg a \in B(a)$.

(ii) It is clear by Lemma 4.6 part $(i)$.

(iii) By $\left(g_{6}\right)$, we can obtain

$$
\begin{aligned}
a \rightarrow b & =1 \rightarrow(a \rightarrow b), \\
& =(a \vee \neg a) \rightarrow(a \rightarrow b), \\
& =[a \rightarrow(a \rightarrow b)] \wedge[\neg a \rightarrow(a \rightarrow b)], \\
& =[a \rightarrow(a \rightarrow b)] \wedge 1, \\
& =a \rightarrow(a \rightarrow b) .
\end{aligned}
$$

(iv) By $\left(e_{7}\right),\left(e_{14}\right)$ and $\left(g_{4}\right)$ we have

$$
\begin{aligned}
a \rightarrow(b \rightarrow c) & \leq a \rightarrow[(a \rightarrow b) \rightarrow(a \rightarrow c)], \\
& =(a \rightarrow b) \rightarrow[a \rightarrow(a \rightarrow c)], \\
& =(a \rightarrow b) \rightarrow(a \rightarrow c) .
\end{aligned}
$$

Thus by $\left(e_{21}\right)$, we can obtain $a \rightarrow(b \rightarrow c)=(a \rightarrow b) \rightarrow(a \rightarrow c)$.

(v) By $\left(e_{15}\right)$ and Lemma 4.6 part (ii), we have:

$$
\begin{aligned}
(a \rightarrow b) \rightarrow b & \leq(b \rightarrow a) \rightarrow[(a \rightarrow b) \rightarrow a], \\
& =(b \rightarrow a) \rightarrow \widetilde{a}, \\
& =(b \rightarrow a) \rightarrow a .
\end{aligned}
$$

(vi) We obtain by $(i v)$ :

$$
\begin{aligned}
a \rightarrow \neg \neg b & =a \rightarrow(\neg b \rightarrow 0), \\
& =(a \rightarrow \neg b) \rightarrow(a \rightarrow 0), \\
& =(a \rightarrow(b \rightarrow 0)) \rightarrow \neg a, \\
& =[(a \rightarrow b) \rightarrow \neg a] \rightarrow \neg a .
\end{aligned}
$$

(vii) It is clear by Lemma 4.6 part (iii) and $\left(g_{7}\right)$.

Corollary 3.8. Let $\xi$ be a good blEQ-algebra and $a, b \in B(E)$. Then

(i) $(a \rightarrow b) \rightarrow b=(b \rightarrow a) \rightarrow a$,

(ii) $(a \rightarrow b) \rightarrow b=\neg a \rightarrow b$. 
Proof. (i) It follows from Lemma 4.7 part $(v)$.

(ii) By $\left(e_{15}\right)$ and Lemma 4.7 part (ii) we obtain

$$
\begin{aligned}
\neg a \rightarrow b & \leq(b \rightarrow a) \rightarrow(\neg a \rightarrow a), \\
& =(b \rightarrow a) \rightarrow a, \\
& =(a \rightarrow b) \rightarrow b .
\end{aligned}
$$

We also have $\neg a \leq a \rightarrow b$ and so $(a \rightarrow b) \rightarrow b \leq \neg a \rightarrow b$. Therefore $(a \rightarrow b) \rightarrow b=\neg a \rightarrow b$.

Lemma 3.9. Let $\xi$ be an blEQ-algebra and $a \in B(E)$. Then we have

(i) $a \rightarrow(a \rightarrow b)=\widetilde{a \rightarrow b}$, for all $b \in E$. Moreover $a \rightarrow \neg a=\widetilde{\neg a}$,

(ii) $a \rightarrow b \leq \widetilde{\neg a \vee b}$, for all $b \in E$,

(iii) $\widetilde{a}=\neg b$, for all $b \in B(a)$,

(iv) If $\xi$ is a good, then $a \rightarrow b=\neg a \vee b$, for all $b \in E$.

Proof. (i) By Lemma 4.3 part (iii) we have:

$$
\begin{aligned}
a \rightarrow(a \rightarrow b) & \leq(a \vee \neg a) \rightarrow[\neg a \vee(a \rightarrow b)], \quad b y\left(l_{4}\right) \\
& =1 \rightarrow(a \rightarrow b), \quad b y\left(e_{7}\right) \\
& =\widetilde{a \rightarrow b} .
\end{aligned}
$$

On the other hand, since $a \leq 1$ we can obtain

$$
\widetilde{a \rightarrow b}=1 \rightarrow(a \rightarrow b) \leq a \rightarrow(a \rightarrow b) .
$$

Therefore $a \rightarrow(a \rightarrow b)=\widetilde{a \rightarrow b}$.

(ii) By Lemma 4.3 part (iii) we have

$$
a \rightarrow b \leq(a \vee \neg a) \rightarrow(\neg a \vee b)=1 \rightarrow(\neg a \vee b)=\widetilde{\neg a \vee b}
$$

(iii) Let $b \in B(E)$. Then

$$
\neg b=b \rightarrow 0 \leq b \rightarrow a \leq(b \vee a) \rightarrow a=\widetilde{a} .
$$

Therefore by Lemma 4.3 part $(v), \neg b=\widetilde{a}$.

(iv) By (ii) we get that $a \rightarrow b \leq \neg a \vee b$ for $b \in E$. Now $b \leq a \rightarrow b$ and $\neg a \leq a \rightarrow b$ imply $\neg a \vee b \leq a \rightarrow b$. Therefore $a \rightarrow b=\neg a \vee b$.

In a $b E Q$-algebra $\xi$, we denote $B^{*}(E):=\{a \in E:<a>\cap<\neg a>=\{1\}\}$. By Lemma 3.4 for all $a, b, c \in E, a \in B^{*}(E)$ iff $a \rightarrow c=\neg a \rightarrow c=1$ implies $c=1$.

Lemma 3.10. Let $\xi$ be an EQ-algebra. Then $\xi$ is semi-separated iff $0,1 \in B^{*}(E)$ iff $B^{*}(E) \neq$ $\emptyset$.

Proof. Let $\xi$ be semi-separated. Then by Lemma $2.7,<1>=\{1\}$ and we get that 


$$
<0>\cap<\neg 0>=<0>\cap<1>=E \cap\{1\}=\{1\},
$$

and

$$
<1>\cap<\neg 1>=\{1\} \cap<\neg 1>=\{1\}
$$

Thus $0,1 \in B^{*}(E)$.

Let $0,1 \in B^{*}(E)$ and $a \sim 1=1$. Then $1 \rightarrow a=1$ and so $a \in<1>\cap<0>=<\neg 0>\cap<$ $0>=\{1\}$. Therefore $a=1$, i.e $E$ is semi-separated.

Now, let $\emptyset \neq B^{*}(E)$. Then there exists $a \in E$ such that $\left.\langle a\rangle \cap<\neg a\right\rangle=\{1\}$.

Thus $a, \neg a \leq 1$ implies $<1>\subseteq<a>\cap<\neg a>=\{1\}$. Therefore $<1>=\{1\}$, so by Lemma 2.7, E is semi-separated.

By the following theorem, we show that $B(E)=B^{*}(E)$, where $\xi$ is a good $b \ell E Q$-algebra.

Theorem 3.11. Let $\xi$ be a good blEQ-algebra. Then $a \in B(E)$ iff $a \in B^{*}(E)$.

Proof. Let $a \in B(E)$. Then by Lemma 4.3, we have

$$
\begin{aligned}
a \vee \neg a=1 & \Rightarrow<a \vee \neg a>=<1>, \\
& \Rightarrow<a>\cap<\neg a>=\{1\}, \\
& \Rightarrow a \in B^{*}(E) .
\end{aligned}
$$

Now, let $a \in B^{*}(E)$. Since $a, \neg a \leq(a \vee \neg a)$ we have $a \rightarrow(a \vee \neg a)=\neg a \rightarrow(a \vee \neg a)=1$, so by Lemma 3.4, we can conclude $a \vee \neg a=1$. Thus $a \in B(E)$.

By the following theorem, we display $B(E)$ is closed under $\wedge, \vee, \rightarrow$ on every good $b \ell E Q$ algebra.

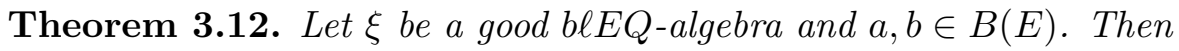

(i) $a \wedge b \in B(E)$ and also $a \wedge b=\neg(a \rightarrow \neg b)$,

(ii) $a \vee b \in B(E)$, moreover $a \rightarrow b \in B(E)$.

Proof. (i) Let $a, b \in B(E)$. Then by Theorem 4.11,

$$
<a>\cap<\neg a>=\{1\} \text { and }<b>\cap<\neg b>=\{1\} .
$$

Also, we have $\langle a>,<b>\subseteq<a \wedge b>$ and $<\neg(a \wedge b)>\subseteq<\neg a>,<\neg b>$. Thus

$$
<\neg(a \wedge b)>\cap<a>\subseteq<\neg a>\cap<a>=\{1\}
$$

and

$$
<\neg(a \wedge b)>\cap<b>\subseteq<\neg b>\cap<b>=\{1\} .
$$

So $<\neg(a \wedge b)>\cap<a>=\{1\}$ and $<\neg(a \wedge b)>\cap<b>=\{1\}$. 
Since $E$ is an $\ell E Q$-algebra, by Theorem 2.7 part (iii) we have:

$$
\begin{aligned}
\{1\} & =[<\neg(a \wedge b)>\cap<a>] \vee[<\neg(a \wedge b)>\cap<b>], \\
& =<\neg(a \wedge b)>\cap[<a>\vee<b>], \\
& =<\neg(a \wedge b)>\cap[<a \wedge b>] .
\end{aligned}
$$

and we conclude that $a \wedge b \in B(E)$.

Also by Lemma 4.7 part (vii), Lemma 4.9 part (iv) and $\left(g_{6}\right)$, we have

$$
\begin{aligned}
a \wedge b & =(\neg \neg a) \wedge(\neg \neg b), \\
& =\neg(\neg a \vee \neg b), \\
& =\neg(a \rightarrow \neg b) .
\end{aligned}
$$

(ii) We have $\langle a \vee b>\subseteq \subset a>$, so $\langle a \vee b>\cap<\neg a>\subseteq<a>\cap<\neg a>=\{1\}$ that is $<a \vee b>\cap<\neg a>=\{1\}$. Similarly $<(a \vee b)>\cap<\neg b>=\{1\}$.

By hypothesis, by Theorem 2.7 part (iii) we get that

$$
\begin{aligned}
\{1\} & =[<a \vee b>\cap<\neg a>] \vee[<a \vee b>\cap<\neg b>], \\
& =<a \vee b>\cap[<\neg a>\vee<\neg b>], \\
& =<a \vee b>\cap[<\neg a \wedge \neg b>], \\
& =<a \vee b>\cap<\neg(a \vee b)>.
\end{aligned}
$$

Therefore $a \vee b \in B(E)$. So by Lemma 4.9 part $(i v)$, we can say $a \rightarrow b \in B(E)$.

The following lemma shows that $B(E)$ has distributive property in a good $b l E Q$-algebra $E$.

Lemma 3.13. Let $\xi$ be a good blEQ-algebra and $a, b, c \in B(E)$. Then $a \vee(b \wedge c)=(a \vee b) \wedge(a \vee c)$.

Proof. By Theorem 4.12 and Lemma 4.9 part (iv), we have

$$
\neg[(\neg a \rightarrow b) \rightarrow \neg(\neg a \rightarrow c)]=(a \vee b) \wedge(a \vee c)
$$

and

$$
\neg a \rightarrow \neg(b \rightarrow \neg c)=a \vee(b \wedge c)
$$


By using $\left(e_{6}\right)$, Lemma 4.6 part (ii) and Lemma 4.7 parts $(i)$ and $(i v)$, we obtain

$$
\begin{aligned}
\neg a \rightarrow \neg(b \rightarrow \neg c) & =[\neg a \rightarrow(b \rightarrow \neg c)] \rightarrow \neg \neg a, \\
& =[(\neg a \rightarrow b) \rightarrow(\neg a \rightarrow \neg c)] \rightarrow \neg \neg a, \\
& =[(\neg a \rightarrow b) \rightarrow((\neg a \rightarrow c) \rightarrow(\neg a \rightarrow 0))] \rightarrow \neg \neg a, \\
& =[(\neg a \rightarrow b) \rightarrow((\neg a \rightarrow c) \rightarrow \neg a) \rightarrow((\neg a \rightarrow c) \rightarrow 0))] \rightarrow \neg \neg a, \\
& =[(\neg a \rightarrow b) \rightarrow(\neg a \rightarrow((\neg a \rightarrow c) \rightarrow 0))] \rightarrow \neg \neg a, \\
& =[[(\neg a \rightarrow b) \rightarrow \neg a] \rightarrow[(\neg a \rightarrow b) \rightarrow \neg(\neg a \rightarrow c)]] \rightarrow \neg \neg, \\
& =[\neg a \rightarrow[(\neg a \rightarrow b) \rightarrow \neg(\neg a \rightarrow c)]] \rightarrow \neg \neg a, \\
& =[\neg a \rightarrow[(\neg a \rightarrow b) \rightarrow \neg(\neg a \rightarrow c)]] \rightarrow(\neg a \rightarrow 0), \\
& =\neg a \rightarrow[[(\neg a \rightarrow b) \rightarrow \neg(\neg a \rightarrow c)] \rightarrow 0], \\
& \geq \neg[(\neg a \rightarrow b) \rightarrow \neg(\neg a \rightarrow c)] .
\end{aligned}
$$

So $(a \vee b) \wedge(a \vee c) \leq a \vee(b \wedge c)$ and also we have $a \vee(b \wedge c) \leq(a \vee b) \wedge(a \vee c)$. Therefore $a \vee(b \wedge c)=(a \vee b) \wedge(a \vee c)$, for all $a, b, c \in B(E)$

\begin{tabular}{|c|c|c|c|c|c|c|c|c|c|c|c|c|c|c|c|c|c|}
\hline$\otimes$ & 0 & $a$ & $b$ & $c$ & $d$ & $e$ & $f$ & 1 & $\sim$ & 0 & $a$ & $b$ & $c$ & $d$ & $e$ & $f$ & 1 \\
\hline 0 & 0 & 0 & 0 & 0 & 0 & 0 & 0 & 0 & 0 & 1 & $e$ & $f$ & $d$ & $e$ & $a$ & $b$ & 0 \\
\hline$a$ & 0 & 0 & 0 & 0 & 0 & 0 & 0 & $a$ & $a$ & $e$ & 1 & $d$ & $f$ & $c$ & $a$ & $c$ & $a$ \\
\hline$b$ & 0 & 0 & 0 & 0 & 0 & 0 & 0 & $b$ & $b$ & $f$ & $d$ & 1 & $e$ & $c$ & $c$ & $b$ & $b$ \\
\hline$c$ & 0 & 0 & 0 & 0 & $a$ & $a$ & $a$ & $c$ & $c$ & $d$ & $f$ & $e$ & 1 & $c$ & $c$ & $c$ & $c$ \\
\hline$d$ & 0 & 0 & 0 & 0 & $d$ & $d$ & $d$ & $d$ & $d$ & $c$ & $c$ & $c$ & $c$ & 1 & $f$ & $e$ & $d$ \\
\hline$e$ & 0 & 0 & 0 & 0 & $d$ & $e$ & $d$ & $e$ & $e$ & $a$ & $a$ & $c$ & $c$ & $f$ & 1 & $d$ & $e$ \\
\hline$f$ & 0 & 0 & 0 & 0 & $d$ & $d$ & $d$ & $f$ & $f$ & $b$ & $c$ & $b$ & $c$ & $e$ & $d$ & 1 & $f$ \\
\hline 1 & 0 & $a$ & $b$ & $c$ & $d$ & $d$ & $f$ & 1 & 1 & 0 & $a$ & $b$ & $c$ & $d$ & $e$ & $f$ & 1 \\
\hline
\end{tabular}

Definition 3.14. An lEQ-algebra $\xi$ is called $(\otimes, \vee)$-distributive if the following equality holds for all $a, b, c \in E$ :

$$
c \otimes(a \vee b)=(c \otimes a) \vee(c \otimes b)
$$

It is easy to check that any chain $E Q$-algebra and residuated $E Q$-algebra are $(\otimes, \vee)$ distributive.

The following examples show that an $E Q$-algebra may not be $(\otimes, \vee)$-distributive in general.

Example 3.15. (i) Consider $E=\{0, a, b, c, d, e, f, 1\}$ such that $0<a<c<d<e<1$ and $0<b<c<d<f<1$. Multiplication and fuzzy equality on $E$ are defined below. It is clear that $(E, \wedge, \otimes, \sim, 1)$ is an blEQ-algebra [11].

We have $c=c \otimes(e \vee f) \neq(c \otimes e) \vee(c \otimes f)=a$, so $E$ is not $(\otimes, \vee)$-distributive.

(ii) Consider the blEQ-algebra $E=\{0, a, b, c, 1\}$ in Example 4.1 part (ii). It is clear that $E$ is neither good and nor $(\otimes, \vee)$-distributive, since $a=a \otimes(c \vee b) \neq(a \otimes c) \vee(a \otimes b)=0$. 
(iii) Consider $E=\{0, a, b, c, d, 1\}$ such that $0<a<b, c<1$ and $0<d<c<1$. Multiplication and fuzzy equality on $E$ are defined below.

\begin{tabular}{|c|c|c|c|c|c|c|c|c|c|c|c|c|c|}
\hline$\otimes$ & 0 & $a$ & $b$ & $c$ & $d$ & 1 \\
\hline 0 & 0 & 0 & 0 & 0 & 0 & 0 \\
\hline$a$ & 0 & 0 & 0 & 0 & 0 & $a$ \\
\hline$b$ & 0 & 0 & $a$ & 0 & 0 & $b$ \\
\hline$c$ & 0 & 0 & 0 & 0 & 0 & $c$ \\
\hline$d$ & 0 & 0 & 0 & 0 & 0 & $d$ \\
\hline 1 & 0 & $a$ & $b$ & $c$ & $d$ & 1 \\
\hline 0 & 1 & 1 & 1 & 1 & 1 & 1 \\
\hline$a$ & 1 & 1 & 1 & 1 & 1 & 1 \\
\hline$b$ & 1 & 1 & 1 & 1 & 1 & 1 \\
\hline$c$ & 1 & 1 & 1 & 1 & 1 & 1 \\
\hline$d$ & 1 & 1 & 1 & 1 & 1 & 1 \\
\hline 1 & 1 & 1 & 1 & 1 & 1 & 1 \\
\hline
\end{tabular}

We can check that $(E, \wedge, \otimes, \sim, 1)$ is an blEQ-algebra and $B(E)=\{0,1, b, d\}$. E is not $(\otimes, \vee)$ distributive and also $B(E)$ is not a Boolean algebra since $b \otimes b=a \notin B(E)$.

Lemma 3.16. Let $\xi$ be $a(\otimes, \vee)$-distributive $E Q$-algebra and $a \in B(E)$. Then for all $x, c \in E$ it holds that

(i) $a^{2}=a$,

(ii) $a \otimes x=a \wedge x$,

(iii) $a \sim b \in B(E)$, for all $b \in B(E)$,

(iv) $a \rightarrow(a \rightarrow c) \leq a \rightarrow \widetilde{c}^{4}$,

$(v)<a>=\left\{x \in E: a \leq \widetilde{x}^{n}\right.$, for some $\left.n \geq 1\right\}$.

Proof. Consider $a \in B(E)$. Then there exists $b \in E$ such that $a \vee b=1$ and $a \wedge b=0$. So we have:

$(i)$

$$
\begin{aligned}
a & =a \otimes 1, \\
& =a \otimes(a \vee b), \\
& =a^{2} \vee(a \otimes b), \\
& =a^{2} .
\end{aligned}
$$

(ii) Since $(a \wedge x) \otimes a \leq a^{2} \wedge(a \otimes x) \leq(a \otimes x)$ and $(a \wedge x) \otimes b \leq(a \otimes b) \wedge(x \otimes b)=0$ we can say

$$
\begin{aligned}
a \wedge x & =(a \wedge x) \otimes 1 \\
& =(a \wedge x) \otimes(a \vee b), \\
& =[(a \wedge x) \otimes a] \vee[(a \wedge x) \otimes b], \\
& \leq(a \otimes x) .
\end{aligned}
$$

Thus $(a \wedge x)=(a \otimes x)$.

(iii) It is clear by $\left(e_{20}\right),($ ii $)$ and Theorem 4.12 part $(i)$.

(iv) It is clear by $\left(e_{19}\right)$. 
(v) Put $T=\left\{x \in E: a \leq \widetilde{x}^{n}\right.$, for some $\left.n \geq 1\right\}$. Let $x \in<a>$. Then there exists $n \geq 1$ such that $a \rightarrow^{n} x=1$. So by $(i v)$, we can find $m \geq 1$ such that $1=a \rightarrow^{n} x \leq a \rightarrow \widetilde{x}^{m}$, that is $a \rightarrow \widetilde{x}^{m}=1$. Hence by $\left(e_{9}\right)$, we obtain $a=a \otimes\left(a \rightarrow \widetilde{x}^{m}\right) \leq \widetilde{x}^{m+1}$. Therefore we conclude $x \in T$.

Now, Let $x \in T$. Then there exists $n \geq 1$ such that $a \leq \widetilde{x}^{n}$, so $a \rightarrow \widetilde{x}^{n}=1$. Thus by $\left(e_{6}\right)$, we can say $1=a \rightarrow \widetilde{x}^{n} \leq a \rightarrow^{n+1} x$. Therefore $a \rightarrow^{n+1} x=1$, that is $x \in<a>$.

By Theorem 4.12 part $(i)$ and Lemma 4.16 part $(i i)$ we have the following result.

Corollary 3.17. Let $\xi$ be a good and $(\otimes, \vee)$-distributive blEQ-algebra and $a, b \in B(E)$. Then $a \otimes b \in B(E)$.

By Theorem 4.12, Lemma 4.16 and Corollary 4.17 we have:

Theorem 3.18. Let $\xi$ be a good and $(\otimes, \vee)$-distributive blEQ-algebra. Then the set $B(E)$ is the universe of a Boolean subalgebra of $E$.

\section{Conclusion}

In this paper, we carried out a study of $E Q$-algebras. We introduced the notion of Boolean center in a lattice ordered $E Q$-algebra $\xi$ with bottom element 0 and showed this set is not empty in general. We also proved some properties of an $E Q$-algebra and using those properties, we studied the properties of a boolean element of $\xi$. We determined that by special type of an $E Q$-algebra with a bottom element, complements of the element are unique. Furthermore, we concluded that Boolean center is characterized under some conditions. Finally, we showed that the Boolean center can be the universe of a Boolean subalgebra of $E$.

The following questions can be raised:what is the relationship between the lattice $(C O N(E))$ of the congruences of $E$ and the lattice $(C O N(B(E)))$ of the congruences of the Boolean algebra $B(E)$ ? What are the topological properties of $B(E)$ ? What properties does $B^{*}(E)$ have, where $E$ is semi-separated? How can we define Boolean prefilters in an $E Q$-algebra and what are their properties? These questions could be topics for further research.

\section{REFERENCES}

[1] C.C. Chang, Algebraic analysis of many valued logics, Trans Am Math Soc 88 (1958) 467-490.

[2] M. El-Zekey, Representable good EQ-algebras, Soft Computing, 14 (2010) 1011-1023.

[3] M. El-Zekey, V. Novák, R. Mesiar, On good EQ-algebras, Fuzzy sets and systems, 178 (2011) 1-23.

[4] F. Esteva, L. Godo, Monoidal t-norm based logic: towards a logic for left-continuous t-norms, Fuzzy Sets Systems, 124 (2001) 271-288.

[5] G. Georgescu, L. Leustean, C. Muresan, Maximal residuated lattices with lifting Boolean center, Algebra Universalis (2010) 63(1) 83-99.

[6] J. Gispert, A. Torrens, Boolean representation of bounded BCK-algebras, Soft Comput 12 (2008) 941-954.

[7] N. Mohtashamnia, L. Torkzadeh, The lattice of prefilters of an EQ-algebra, Fuzzy Sets and Systems, 311 (2017) 86-98. 
[8] P. Hájek, Metamathematics of Fuzzy Logic, Kluwer, Dordrecht (1998).

[9] L. Z. Liu, K.T. Li, $R_{0}$-algebras and weak dually residuated lattice ordered semigroups, Czech Math J, 56 (2006) 339-348.

[10] V. Novák, EQ-algebras:primary concepts and properties, in: Proc. Czech-Japan Seminar, Ninth Meeting. Kitakyushu and Nagasaki, Graduate School of Information, Waseda University, August (2006) 18-22.

[11] V. Novák, B. De Baets, EQ-algebra, Fuzzy sets and systems, 160 (2009) 2956-2978.

[12] L. A. Zadeh, Is there a need for fuzzy logic? Inform Science, 178 (2008) 2751-2779.

[13] H. J. Zhou and B. Zhao, Stone-like representation theorems and three-valued letters in R $R_{0}$-algebras (nilpotent minimum algebras), Fuzzy Sets Systems, 162 (2011) 1-26.

\section{Lida Torkzadeh}

Departement of Mathematics, Kerman Branch Islamic Azad university, Kerman,Iran.

ltorkzadeh@yahoo.com

\section{Neda Mohtashamnia}

Departement of Mathematics, Kerman Branch Islamic Azad university, Kerman,Iran.

nmohtashamniya@yahoo.com 